\title{
Two-photon interference with true thermal light
}

\author{
Yan-Hua Zhai ${ }^{1}$, Xi-Hao Chen ${ }^{1,2}$, Da Zhang ${ }^{1}$, Ling-An $\mathrm{Wu}^{1, *}$ \\ ${ }^{1}$ Institute of Physics, CAS, Beijing 100080, China \\ ${ }^{2}$ Department of Physics, Liaoning University, Shenyang 110036, China
}

\begin{abstract}
Two-photon interference and "ghost" imaging with entangled light have attracted much attention since the last century because of the novel features such as non-locality and sub-wavelength effect. Recently, it has been found that pseudo-thermal light can mimic certain effects of entangled light. We report here the first observation of two-photon interference with true thermal light.
\end{abstract}

PACS numbers: $42.50 . \mathrm{Dv}, 42.25 . \mathrm{Hz}, 42.50 . \mathrm{St}$

Interference is described in textbooks as the coherent superposition of waves, and the ability to interfere is regarded as a special attribute, known as coherence, of the radiation source. Optical coherent sources include lasers high above threshold, but the majority of light sources such as thermal light are incoherent. To describe the intensity of the field from coherent sources the amplitudes from all sources present are superposed and summed together before the intensity is calculated, whereas in the case of incoherent sources, the intensities from all the sources can generally be added directly. Apart from the first order field intensities, different sources exhibit different characteristics in their higher order intensity properties, and this has led to widespread studies of the nature of a variety of quantum and classical, coherent and incoherent light sources. Many interesting and important applications have been developed therefrom.

Of particular interest are the second order intensity characteristics, which of course are easier to investigate than the higher orders. Since Hanbury-Brown and Twiss (HBT) 1] first measured the joint-intensity of light, coincidence measurements have been applied to measure the second-order coherence function $\left(G^{(2)}\right)$ of various photon fields. In particular, novel phenomena such as "ghost" imaging [2] and "ghost" interference [3] have been observed through coincidence measurements of the light generated by spontaneous parametric down-conversion (SPDC). In the ghost interference experiment with an SPDC entangled light source, the two-photon amplitudes from both slits are added together as with coherent light sources, although the field would seem to be incoherent from the usual viewpoint. Related to these kinds of phenomena are experiments that demonstrate seemingly nonclassical effects such as sub-wavelength diffraction [4]. At one time it was thought that these phenomena were exclusive to quantum entangled light [5]. However, it has recently been shown theoretically that thermal light can generate similar effects to those of entangled light [6, 7, 8, 9], and experiments to prove this have been performed using pseudo-thermal light 10, 11, 12]. However, in all these experiments the primary light source

\footnotetext{
*Corresponding author: wula@aphy.iphy.ac.cn
}

was a He-Ne laser, the coherent beam being converted to pseudo-thermal light by a rotating ground glass plate or some other means. Different from these previous investigations we report the first two-photon sub-wavelength experiment with true thermal light (sometimes called chaotic light), an incoherent light source exhibiting thermal statistics that cannot interfere in the conventional sense of the term, i.e. does not exhibit first order interference.

We should mention here that the distinction between thermal and chaotic light is not so well defined and different authors give different definitions. In Ref. [13], thermal light is described as the radiation emitted by spontaneous emission from "a large collection of atoms or molecules, excited to high energy states by thermal, electrical, or other means" when they "randomly and independently drop to lower energy states", and the sun, incandescent bulbs and gas discharge lamps are cited as examples. Some authors specify that discharge and filament lamps as well as thermal cavities are forms of chaotic light sources 14, 15], while thermal light is the broad spectral emission from a thermally excited glowing filament 15]. Mandel and Wolf define thermal radiation as "radiation that is derivable from blackbody radiation by any linear filtering process" and point out that "It has sometimes also been called chaotic radiation" [16]. Since a hollow cathode lamp can be portrayed by thermal statistics, we employ the term "thermal light" here in order to correspond and contrast with previous two-photon interference experiments that used pseudo-thermal light.

An outline of the experimental set-up is shown in Fig. [1 We employed a commercial rubidium hollow-cathode lamp [17] manufactured by the General Research Institute for Nonferrous Metals (China), which is the type commonly used in atomic absorption spectroscopy because of its relatively sharp spectral line width. The lamp was powered by a direct current of $20 \mathrm{~mA}$ in our experiments, and the resonance wavelength was $780 \mathrm{~nm}$. The coherence time $\tau_{0}$ was estimated from an HBT type measurement of the second-order correlation function to be about $0.2 \mathrm{~ns}[19]$, which is much shorter than that of previous experiments using randomly scattered light from a He-Ne laser.

In Fig. 1 the light from the lamp is focused by the convex lens $\left(\mathrm{L}_{1}\right)$ of $10 \mathrm{~cm}$ focal length onto a diffraction 


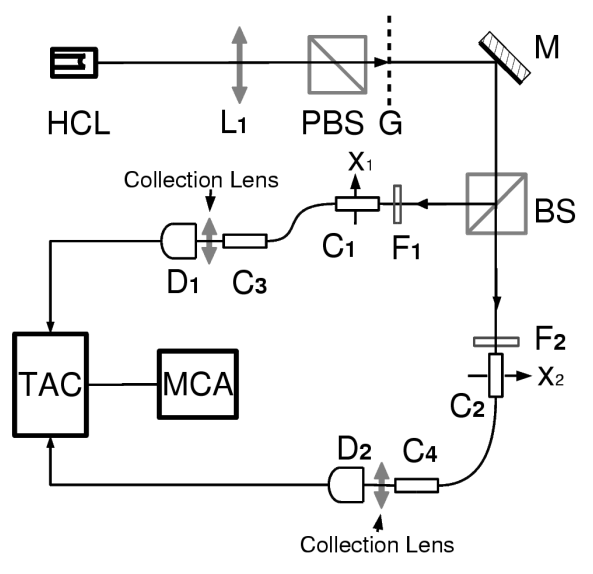

FIG. 1: Experimental set-up. HCL: hollow cathode lamp; $\mathrm{L}_{1}$ : lens of focal length $10 \mathrm{~cm}$; G: grating of groove width $0.08 \mathrm{~mm}$ and groove spacing $0.2 \mathrm{~mm} ; \mathrm{F}_{1}, \mathrm{~F}_{2}$ : interference filters transmitting about $70 \%$ at $780 \mathrm{~nm}$. PBS: polarizing beam splitter; BS: non-polarizing beam splitter; $\mathrm{C}_{1}-\mathrm{C}_{4}$ : fiber collimators. Effective diameter of fiber collimators in front of the detectors is $2 \mathrm{~mm}$.

grating $(\mathrm{G})$ to form a secondary light source. A polarizing beam splitter (PBS) just before the grating allows only the horizontally polarized component of the incoherent beam to pass. The inner diameter of the hollow cathode is $3 \mathrm{~mm}$, from which its image on the grating is calculated to be about $1 \mathrm{~mm}$ in diameter. The width $b$ of a grating groove is $0.08 \mathrm{~mm}$ and the spacing between grooves $d=0.2 \mathrm{~mm}$, so five slits are illuminated. After reflection by a mirror $(\mathrm{M})$ the beam is divided by a 50\%/50\% non-polarizing beam splitter (BS). The reflected and transmitted beams pass through interference filters $F_{1}$ and $F_{2}$ before being coupled into single photon detectors $\mathrm{D}_{1}$ and $\mathrm{D}_{2}$ (Perkin Elmer SPCM-AQR13), respectively, through fiber collimators $\mathrm{C}_{1}$ and $\mathrm{C}_{2}$, and finally collection lenses. Both collimators $\mathrm{C}_{1}$ and $\mathrm{C}_{2}$ can be translated horizontally across the beam. The transmission of the interference filters is about $70 \%$ at $780 \mathrm{~nm}$ and the receiving lens of the collimators about $2 \mathrm{~mm}$ in diameter. The distance $z$ from the grating $(\mathrm{G})$ to either detector is $162 \mathrm{~cm}$. The detector output signals are sent to a time-amplitude converter (TAC), with $\mathrm{D}_{1}$ and $\mathrm{D}_{2}$ providing the "start" and "stop" signals, respectively. The TAC output is connected to a multi-channel analyzer (MCA), and the computer displays a histogram of the different intervals between the times of arrival of the photons at the two detectors. From this we obtain the relation between the photon count rate and time interval $\tau$, and subsequently the second-order correlation function

$$
G^{(2)}(\tau)=\left\langle\hat{E}_{2}(\tau)^{(-)} \hat{E}_{1}(0)^{(-)} \hat{E}_{1}(0)^{(+)} \hat{E}_{2}(\tau)^{(+)}\right\rangle
$$

where $\hat{E}_{i}^{(+)}, \hat{E}_{i}^{(-)}$are the positive and negative frequency field operators at detectors $\mathrm{D}_{i}(i=1,2)$, respectively.

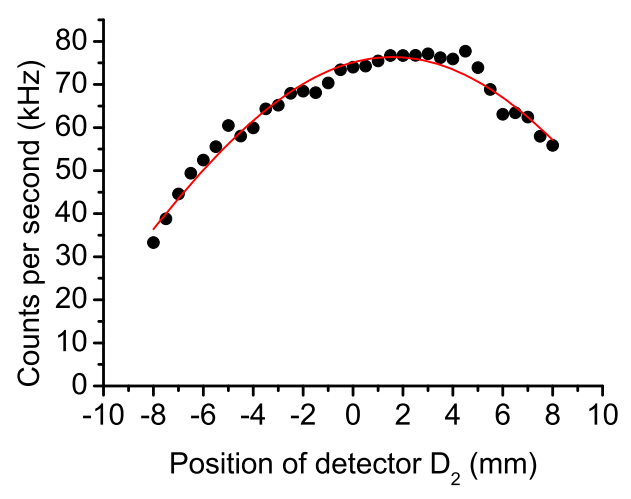

FIG. 2: Single detector counts vs. position of detector $\mathrm{D}_{2}$. The solid curve is a Gaussian fit of data points.

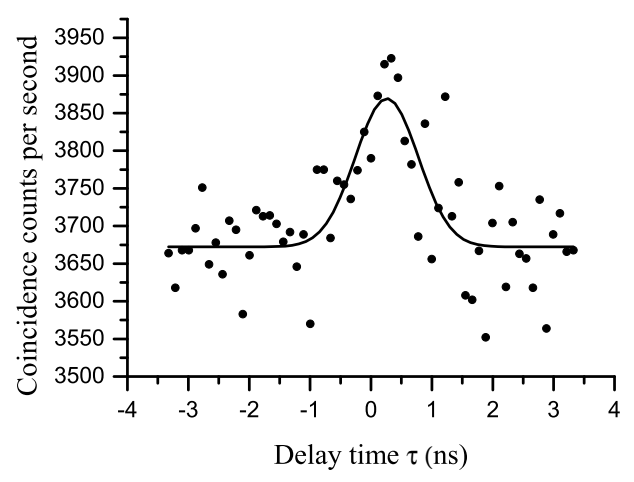

FIG. 3: Joint detection counts vs time interval of the photons detected at $D_{1}$ and $D_{2}$. The solid curve is a Gaussian fit of data points.

To begin with, the detector $\mathrm{D}_{1}$ was kept fixed while $\mathrm{D}_{2}$ (collimator $\mathrm{C}_{2}$ ) was scanned in the horizontal direction and the single counts of $\mathrm{D}_{2}$ recorded as a function of its position. As can be seen from Fig. 2 no first-order interference pattern was observed so there is no first-order coherence in our experiment.

Next, the collimators $\mathrm{C}_{1}$ and $\mathrm{C}_{2}$ were both fixed in the center of their beams at the positions $x_{1}=0, x_{2}=0$, respectively. The distribution of the times of arrival of photons at the detectors as displayed on the MCA is shown in Fig. 3 from which we obtained the value of $g^{(2)}$ [19] by dividing the values of the average $G^{(2)}\left(t_{2}-t_{1}\right)$ for $|\tau| \leq 0.25 n s$ (corresponding to signals arriving almost simultaneously) by the value of $G^{(2)}(\tau)$ for $|\tau| \gg 1.3 \mathrm{~ns}$ (corresponding to signals arriving randomly well beyond any correlation times), i.e.

$$
g^{(2)}=\frac{G^{(2)}(|\tau| \leq 0.25 \mathrm{~ns})}{G^{(2)}(|\tau| \geq 1.3 \mathrm{~ns})} .
$$

With $\mathrm{C}_{1}$ still fixed at $x_{1}=0, \mathrm{C}_{2}$ was then moved in steps of $0.5 \mathrm{~mm}$ or $1 \mathrm{~mm}$ through $x_{2}= \pm 10 \mathrm{~mm}$. From the 


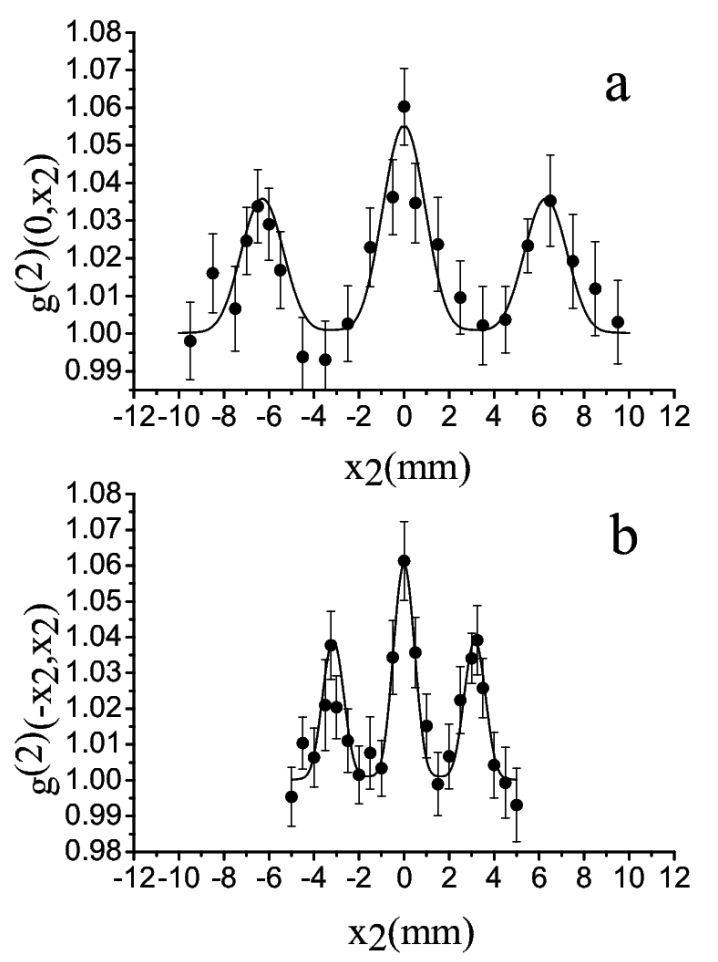

FIG. 4: Normalized second-order correlation function of thermal light (a) $g^{(2)}\left(0, x_{2}\right)$ vs position of detector $\mathrm{D}_{2}$ with detector $\mathrm{D}_{1}$ fixed. (b) $g^{(2)}\left(-x_{2}, x_{2}\right)$ vs position of detector $\mathrm{D}_{2}$. The solid curve is calculated taking into consideration the finite size of the detectors [21]

data obtained on the MCA the normalized second-order correlation function $g^{(2)}$ was calculated, and the secondorder interference-diffraction pattern of the grating plotted, as shown in Fig. 四. We see that the pattern looks classical, with a distance of about $6.5 \mathrm{~mm}$ between the zero and first order interference peaks. This agrees well with the calculated value of $6.3 \mathrm{~mm}$ obtained from the grating equation for first-order interference-diffraction of the field seen at a single detector [20].

$$
\sin \theta-\sin \theta_{0}=m \lambda / d \quad(m=0, \pm 1, \pm 2, \cdots),
$$

where $\theta_{0}$ is the angle of the incident light and $\theta$ that of the diffracted light measured from the normal to the grating plane. The integer $m$ represents the path difference in wavelengths between light diffracted in the direction of the maximum, from corresponding points in two neighboring grooves.

When the fiber collimators $\mathrm{C}_{1}$ and $\mathrm{C}_{2}$ were scanned in opposite directions $(x,-x)$ in steps of $0.25 \mathrm{~mm}$ or $0.5 \mathrm{~mm}$ simultaneously, the second-order interference-diffraction pattern of the grating shown in Fig. 4 $4 \mathrm{~b}$ was obtained. The distance from the zero order to the first order interference peak is about $3.25 \mathrm{~mm}$, which is exactly half that of the classical case. This is the well known subwavelength effect first predicted and observed for two-

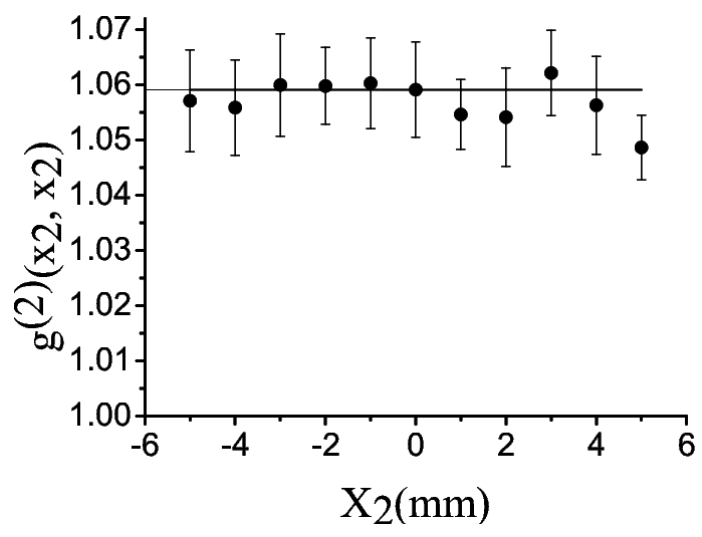

FIG. 5: Normalized second-order correlation function $g^{(2)}\left(x_{2}, x_{2}\right)$ of thermal light vs position of detector $\mathrm{D}_{2}$.

photon interference with entangled photon pairs [4].

However, when both collimators were scanned simultaneously in the same direction $(x, x)$, no interference pattern was observed, as shown in Fig. 5 This is different from the case with an entangled light source [4].

In our experiment, because the coherence time of the thermal light source is shorter than the time resolution of the detection system which is about $1 \mathrm{~ns}$, and the detectors are not point-like, the maximum of $g^{(2)}$ cannot reach 2 and the visibility is only about $3 \%[19,22$.

These experimental results are in good accordance with the values predicted theoretically [6, 7, 8$]$ for the detectors scanned in opposite directions,

$$
g^{(2)}(x,-x)-1 \propto \operatorname{sinc}^{2}\left[\frac{\pi b x}{(\lambda / 2) z}\right] \cos ^{2}\left[\frac{\pi d x}{(\lambda / 2) z}\right]
$$

and for the detectors scanned in the same direction,

$$
g^{(2)}(x, x) \propto \text { const. }
$$

To summarize, two-photon interference with subwavelength fringes has been observed for the first time with true thermal light. Although the visibility is low compared with entangled two-photon interference, which exhibits high visibility but low intensity, thermal light is of course much easier to generate and measure than entangled light. The constant background in the interference-diffraction pattern, an unavoidable feature of thermal light sources, could nevertheless be removed by some means, e.g. digitally.

It is interesting to note that for incoherent light sources that do not exhibit first order interference in the plane of detection, we can generally just sum the intensities produced by the individual sources, instead of having to sum the field amplitudes. However, for second order correlation measurements of the field at two distinct space-time locations, we cannot merely add the intensities even for incoherent thermal fields. It is the individual field amplitude components that have to be summed, and it is this that gives rise to the intensity product term of the HBT 
experiment, and two-photon interference features. What is the difference, or similarity, between classical and entangled light sources? It is evident that the two-photon interference and imaging effects observed so far both originate in the correlation of the photons arriving at the detectors. For entangled sources the strong one-to-one correspondence of the two photons within each pair allows a theoretical visibility of $100 \%$ for both ghost imaging and ghost interference. For classical thermal sources it is the bunching effect that produces the weak but finite correla- tion. These are all consequences of the photon statistics, which can give rise to many rich and varied phenomena.

We are grateful to Yan-Hua Shih, De-Zhong Cao, KaiGe Wang, and Shi-Yao Zhu for useful discussions. We also thank Zhan-Chun Zuo and Hai-Qiang Ma for their experimental assistance. This work was supported by the National Natural Science Foundation of China (Grant No. 60178013), the National Program for Basic Research in China (Grant 001CB309301), and the Knowledge Innovation Program of the Chinese Academy of Sciences.
[1] R. H. Brown, R. Q. Twiss. Nature. 177, 27(1956); Nature. 178, 1046(1956).

[2] T. B. Pittman, Y. H. Shih, D. V. Strekalov, and A. V.Sergienko, Phys. Rev. A 52, R3429 (1995).

[3] D. V. Strekalov, A. V. Sergienko, D. N. Klyshko, and Y. H. Shih, Phys. Rev. Lett. 74, 3600(1995).

[4] M. D'Angelo, M. V. Chekhova, and Y. H. Shih, Phys. Rev. Lett. 87, 013602-1(2001).

[5] A. F. Abouraddy, B. E. A. Saleh, ${ }^{*}$ Alexander V. Sergienko, and M. C. Teich. Phys. Rev. Lett. 87, 1236021 (2001).

[6] Kaige Wang and De-Zhong Cao. Phys. Rev. A. 70, 041801(R) ( 2004).

[7] A. Gatti, E. Brambilla, M. Bache, and L. A. Lugiato. Phys. Rev. A. 70, 013802 (2004).

[8] Yangjian Cai, Shi-Yao Zhu. Opt. Lett. 29,2716(2004).

[9] Jing Cheng, Shen-sheng Han. Phys. Rev. Lett. 92, 093903-1( 2004).

[10] G. Scarcelli, A. Valencia, Y. H. Shih. Phys. Rev. A. 70, 051802(R) (2004); EuroPhysics Letters 68, 618 (2004)

[11] Jun Xiong, De-Zhong Cao, Feng Huang, Hong-Guo Li, Xu-Juan Sun, Kaige Wang. Phys. Rev. Lett. 94, 173601$1(2005)$.

[12] D. Magatti, F. Ferri, A. Gatti, M. Bache, E. Brambilla,
L.A. Lugiato. Phys. Rev. Lett. 94, 183602 (2005).

[13] J. W. Goodman, Statistical Optics (John Wiley \& Sons, New York 1985).

[14] Rodney Loudon, The Quantum Theory of Light (Clarendon Press, Oxford 1983).

[15] H.-A. Bachor and T. C. Ralph, A Guide to Experiments in Quantum Optics (2nd, rev. and enl. ed. Wiley-VCH, c2004).

[16] L. Mandel and E. Wolf, Optical Coherence and Quantum Optics (Cambridge University Press, New York 1995).

[17] Bernhard Welz, Atomic Absorption Spectroscopy ( Verlag Chemie. Weinhem. New York 1976).

[18] R. Hanbury Brown, The Intensity Interferometer. ( Taylor and Francis Ltd. London 1974). See Eq. 4.26.

[19] Da Zhang, Xi-Hao Chen, Yan-Hua Zhai, and Ling-An Wu. Opt. Lett. 30, 2354 (2005).

[20] M. Born and E. Wolf, Principles of Optics (Cambridge University Press, Cambridge ; New York 1999).

[21] R. H. Brown, R. Q. Twiss. Proc. R. Soc. London, Ser. A. 243, 291 (1958).

[22] S. Thanvanthri, M. H. Rubin. Phys. Rev. A 70, 063811 (2004). 